
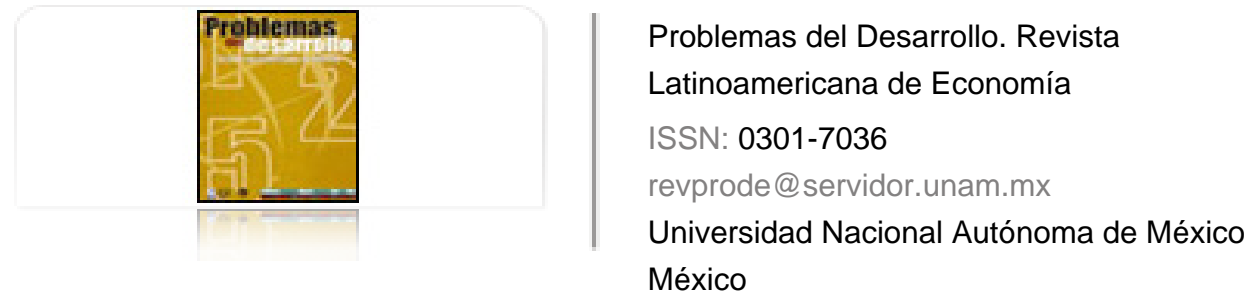

Burgos, Benjamín; Mungaray, Alejandro

Apertura externa, inequidad salarial y calificación laboral en México, 1984-2002

Problemas del Desarrollo. Revista Latinoamericana de Economía, vol. 39, núm. 152, enero-marzo, 2008, pp. 87-111

Universidad Nacional Autónoma de México

Distrito Federal, México

Disponible en: http://www.redalyc.org/articulo.oa?id=11820668006

Cómo citar el artículo

- Número completo

- Más información del artículo

Página de la revista en redalyc.org

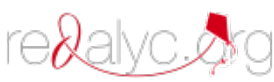

Sistema de Información Científica

Red de Revistas Científicas de América Latina, el Caribe, España y Portugal Proyecto académico sin fines de lucro, desarrollado bajo la iniciativa de acceso abierto 


\title{
APERTURA EXTERNA, INEQUIDAD SALARIAL Y CALIFICACIÓN LABORAL EN MÉXICO, 1984-2002
}

\author{
Benjamín Burgos* \\ Alejandro Mungaray**
}

Fecha de recepción: 2 de julio de 2007. Fecha de aceptación: 28 de octubre de 2007.

\section{Resumen}

En 1985 en México se inició un amplio proceso de liberalización comercial, el cual fue reforzado con el inicio del Tratado de Libre Comercio de América del Norte en 1994. En este artículo se analiza el impacto de la apertura externa sobre el mercado de trabajo y, en particular, la situación de la mano de obra por nivel de calificación, así como la desigualdad salarial. Para ello, a partir de fuentes de datos de personas y empresas, se utilizan diversas metodologías que van desde el cálculo y análisis de indicadores de desigualdad, la estimación de funciones mincerianas de retribuciones hasta regresiones de indicadores de remuneraciones y empleo de trabajadores calificados y no calificados. Como resultado se encontró que con la apertura externa aumentó la inequidad salarial, aunque este proceso no fue lineal y además fue influido por otros factores.

Palabras claves: apertura externa, mercado de trabajo, calificación laboral, retornos a la educación, inequidad salarial.

* Profesor en el Departamento de Economía de la Universidad de Sonora. Correo electrónico: Burgos@guaymas.uson.mx.

** Profesor de Relaciones Internacionales de la Universidad Autónoma de Baja California; además es miembro del SNI y de la AMC. Correo electrónico: mungaray@uabc.mx. 


\section{Summary}

A broad process of trade liberalization began in Mexico in 1985. This was strengthened by the coming into effect of the North American Free Trade Agreement in 1994. This article analyzes the impact on the labor market of the external opening and, in particular, the situation for labor by level of training, as well as inequalities in pay. For this purpose, based on various data sources for individuals and companies, a number of methodologies are used, ranging from the calculation and analysis of inequality indicators, estimating Mincerian functions for salaries, to regressions of indicators for remunerations and employment of skilled and unskilled workers. As a result it was found that the external opening increased wage iniquity, although this process was not linear and was also influenced by other factors.

Key words: external opening, labor market, labor skilling, returns to education, wage iniquity.

\section{Résumé}

En 1985 s'est amorcé au Mexique un ample processus de libéralisation commerciale, lequel fut renforcé par la mise en route du traité de libre échange d'Amérique du Nord en 1994. Dans cet article, il est procédé à l'analyse de l'impact de l'ouverture externe sur le marché de l'emploi et, en particulier, sur la situation de la main d'œuvre selon son niveau de qualification, de même que sur les différences de salaire. Pour se faire, on s'est basé sur des données de particuliers et d'entreprises, lesquelles ont été étudiées selon diverses méthodologies qui vont du calcul et de l'analyse d'indicateurs d'inégalité à l'estimation de fonctions minceriennes de rétributions, passant par des régressions sur les indicateurs de rémunérations et d'emploi de travailleurs qualifiés et non qualifiés. La conclusion de ce travail est que l'ouverture externe a augmenté les différences de salaire, bien que ce processus ne soit pas linéaire et ait été de plus influencé par d'autres facteurs.

Mots clés: ouverture externe, marché de l'emploi, qualification professionnelle, retour à la formation, différences de salaire.

\section{Resumo}

Em 1985 no México iniciou-se um amplo processo de liberalização comercial, o qual foi reforçado com o inicio do Tratado de Livre Comércio da América do Norte em 1994. Neste artigo analisa-se o impacto da abertura externa sobre o mercado de trabalho e, em particular, a situação da mão de obra por nível de qualificação, assim como a desigualdade salarial. Para isso, a partir de fontes de dados de pessoas e empresas, utilizam-se diversas metodologias que vão desde o cálculo e análise de indicadores de desigualdade, a estimação de funções de salários Mincerianas até regressões de indicadores de remunerações y emprego de trabalhadores qualificados $e$ não qualificados. Como resultado encontrou-se que com a abertura externa aumentou a iniquiidade salarial, apesar deste processo não ter sido linear e, além disso, ter sido influenciado por outros fatores.

Palavras-chave: abertura externa, mercado de trabalho, qualificação laboral, retornos à educação, iniqüidade salarial. 


\section{Introducción}

mediados de los años ochenta del siglo pasado, México inició una serie de
reformas tendentes a cambiar el patrón de desarrollo que giraba en torno a una
economía cerrada basada en el modelo sustitutivo de importaciones y sostenida en una amplia participación del Estado en la economía. El primer paso fue eliminar una gran cantidad de barreras comerciales. En tan sólo 30 meses, de junio de 1985 a diciembre de 1987, el valor del producto doméstico cubierto con licencias de importación pasó de $92.2 \%$ a $25.4 \%$ y el promedio ponderado de tarifas se redujo de $23.5 \%$ a 11.8\% (Pacheco-López, 2004:5). En ese mismo periodo el país inicia negociaciones para integrarse al Acuerdo General sobre Aranceles Aduaneros y Comercio (GATT), hoy Organización Mundial de Comercio (OMC), lo cual logra en 1986. Esa situación prevalece hasta 1994 cuando entra en vigor el Tratado de Libre Comercio de América del Norte (TLCAN), el cual implicó una apertura adicional de manera gradual, con fases bien definidas y asimétricas para México.

Si bien los cambios que se dieron en materia de política comercial son importantes, éstos se realizaron como parte de un conjunto de reformas y políticas cuyo objetivo fue transformar de modelo de desarrollo. Entre ellas destacan el proceso de privatización de gran cantidad de empresas estatales; la reducción del gasto público, la racionalización de los subsidios y la tendencia a incrementar los ingresos tributarios para reducir los déficit fiscales; la flexibilización de los controles sobre la inversión extranjera y la liberalización de la cuenta de capital, al eliminarse la mayoría de las restricciones que existían para el ingreso de capitales externos a los mercados financieros mexicanos.

De acuerdo con datos del Banco Mundial (Indicadores de desarrollo mundiales, en línea), el sector externo muestra un crecimiento en el flujo comercial. Las exportaciones que en 1985 se ubicaban en niveles cercanos a los 28,500 millones de dólares, prácticamente se duplicaron para 1993. Con la puesta en marcha del TLCAN se observa un crecimiento mayor, alcanzando en 2002 la cifra de 173,000 millones de dólares, $27 \%$ respecto al Producto Interno Bruto (PIB). De manera análoga se observa un crecimiento significativo en las importaciones, ubicándose en 2002 en niveles de 186,000 millones de dólares, 29\% del PIB.

La inversión extranjera directa (IED) también se vio sensiblemente afectada. Antes de los primeros cambios de 1989 en la legislación respectiva, la IED no rebasaba los 3,000 millones de dólares al año y, por tanto, no se vio fuertemente afectada durante la primer etapa de liberalización comercial. A partir de ese año se fue incrementando gradualmente y desde 1994, con la puesta en marcha del TLCAN, empie- 
zan a entrar grandes flujos que permiten alcanzar un promedio de 13,500 millones de dólares anuales, los cuales tuvieron un impacto importante en el crecimiento de la economía y el empleo durante los primeros años de esta segunda etapa. Por otra parte, la mayor parte de ella se ha dirigido al sector manufacturero, principalmente hacia la actividad maquiladora y, en menor medida, al sector comercio y servicios. En estos últimos destacan los financieros como principales receptores de IED (Pacheco-López y Thirlwall, 2002). Regionalmente también se observa una reorientación de los flujos de IED hacia los estados del norte y centro del país. De los 77,960.8 miles de millones de dólares que ingresaron a México por ese concepto entre 1994 y 2000, 28\% se ubicó en cinco estados fronterizos del norte (Banco de Información Económica, INEGI).

En tal contexto, el objetivo general de este trabajo es analizar el impacto que tuvo la apertura externa sobre el mercado laboral de México, buscando en particular examinar los cambios en el nivel de cantidad y de demanda relativa de trabajo calificado y no calificado y su respectiva remuneración y el efecto sobre la dispersión salarial, tomando en cuenta el periodo de la liberalización comercial comprendido entre 1984 y 2002 (Burgos, 2005). Para ello se trabaja con la hipótesis de que la apertura externa tuvo importantes efectos sobre el mercado de trabajo y, en particular, favoreció la mano de obra calificada y provocó un aumento en la desigualdad salarial, contrario a lo sugerido por el marco analítico de la teoría convencional del comercio internacional. Ello se realiza a partir de un análisis estadístico y econométrico aplicado a las bases de datos de las Encuestas Nacionales de Ingresos y Gastos de los Hogares y los Censos Económicos existentes para el periodo estudiado. En general, se demostró que durante el periodo analizado de apertura externa la inequidad salarial ligada a la liberalización comercial tendió a crecer en el país, si bien dicho proceso no fue lineal y fue influido por otros factores como las crisis económicas, la oferta relativa de mano de obra calificada y la política económica.

Es pertinente aclarar que si bien en el presente estudio se avanza en el análisis de la desigualdad salarial en México - a partir de elementos como el proceso de apertura externa, un periodo de estudio mayor, diversas fuentes de información, el uso de diversas metodologías y su liga con los ritmos de la liberalización comercial— no se examinan otros efectos sobre el mercado laboral. Tampoco se analizan a profundidad los factores que en México podrían incidir en el incumplimiento de los postulados de la teoría convencional del comercio internacional, ni se hace una valoración a fondo de las condiciones de oferta de trabajo calificado y su posible influencia como determinante de la evolución de la inequidad salarial en la región. 


\section{Liberación comercial y distribución del ingreso}

La discusión sobre los efectos del comercio de bienes y servicios entre los diversos países existe desde hace varios siglos, y sus antecedentes se encuentran en los trabajos de Adam Smtih y David Ricardo. En 1933 Bertil Ohlin formalizó el llamado teorema Heckscher-Ohlin, el cual, a diferencia de Ricardo, parte de la existencia de dos factores de la producción (capital y trabajo), que abre la posibilidad de considerar los efectos del comercio sobre la distribución del ingreso. Una conclusión de este modelo es que las distintas dotaciones de factores determinan los precios relativos en las naciones. En aquéllas con abundancia de capital su precio será bajo, mientras que en donde predomine el factor trabajo, los salarios serán menores. Esta diferencia en dotación de recursos tiende a determinar el patrón de comercio internacional, de manera que los gobiernos deben dedicarse a la producción y exportación de bienes en los que tienen ventajas comparativas por la utilización intensiva del factor abundante, típicamente el capital en los países desarrollados y el trabajo en los menos desarrollados.

Con base en un modelo de dos bienes y dos factores, Stolper y Samuelson (1941) relacionan el comercio internacional con la distribución del ingreso. El supuesto es que el mercado de productos en competencia perfecta, con libre entrada de firmas y rendimientos constantes a escala, permite que los bienes tengan un precio de acuerdo con sus costos marginales, garantizando un equilibrio de beneficios cero. Ello permite establecer una relación sistemática entre los precios de producción y los de los factores. Como la liberalización comercial incrementaría el precio de los bienes que utilizan intensivamente el factor abundante de exportación, y con ello su retribución, en un país donde el factor abundante es la mano de obra, la apertura comercial provocaría que la producción se desplazara hacia los bienes que utilizan intensamente ese insumo, mientras que aumentaría la importación de bienes intensivos en el uso del capital, que serían comprados a precios más bajos. Ello provocaría una reducción de la retribución al capital y un aumento en el pago al factor trabajo. Bajo esta lógica se mejoraría la distribución del ingreso. Lo contrario sucedería en países con abundancia relativa de capital.

Como una extensión de este teorema, Grilinches (1969) desarrolla un modelo donde el factor trabajo se divide en calificado y no calificado, el cual permite obtener conclusiones análogas. En los países menos desarrollados con abundancia de trabajo no calificado, la apertura comercial ocasiona una reducción en los precios de los bienes intensivos en uso del trabajo calificado y un aumento de los precios de los bienes que utilizan intensivamente trabajo no calificado. Este cambio en precios relativos provocaría un aumento de la demanda de trabajo no calificado y, por tanto, 
un incremento en sus respectivos salarios, con lo cual las brechas salariales dentro de esas naciones tenderían a cerrarse.

Mientras en algunos países se cumplían las predicciones de la teoría convencional del comercio internacional, en otros se tenían resultados opuestos. Esto ha motivado la búsqueda de otras explicaciones. Una de ellas se basa en el papel de la tecnología, el capital y el trabajo calificado como factores de producción complementarios. En general, se parte de la tendencia al cambio tecnológico sesgado hacia el capital y el conocimiento. Para este enfoque es importante no sólo la liberalización del flujo de bienes, sino también de la inversión y de los mercados financieros. La apertura en la economía ocasiona la introducción de nuevas tecnologías y mejores formas de organización de la producción. La entrada de inversión extranjera directa a los países en desarrollo, con el consiguiente incremento en la importación de maquinaria y equipo, provoca un crecimiento en la demanda de trabajo calificado y con ello un aumento en su retribución, que se manifiesta en una elevación de la desigualdad salarial (Saba, 2001).

Otra explicación a la situación que se presenta en algunos países en desarrollo respecto a las predicciones del teorema Stolper-Samuelson fue realizada por Davis (1996). Su modelo utiliza algunos supuestos compatibles con los de la teoría convencional. Desde este enfoque un país puede tener abundancia del factor trabajo, pero ser abundante en capital en relación con un conjunto de naciones definidas dentro del mismo "cono de diversificación". A partir de ello se obtienen consecuencias en la distribución del ingreso opuestas a las del teorema Stolper-Samuelson. En esta situación, en países menos desarrollados los salarios de los trabajadores calificados tenderán a elevarse en relación con las remuneraciones de los no calificados y la distribución salarial se agravaría. Por el contrario, las naciones que dispongan de abundante mano de obra no calificada verán elevar sus salarios respectivos y con ello reducir la dispersión salarial dentro del país. En el primer caso estarían México y otras naciones latinoamericanas y en el segundo China, India y Pakistán. Feestra y Hanson (1995) argumentan que la inversión extranjera directa y el fenómeno de outsourcing desarrollado por empresas multinacionales traslada desde los países desarrollados la producción de bienes o partes del proceso de producción menos intensivas en conocimiento hacia naciones menos desarrolladas, pero más intensivas en conocimiento para los estándares de estos últimos países. En consecuencia, elevan la demanda relativa de trabajo calificado tanto en la nación de origen como en el país huésped e incrementa la desigualdad salarial en ambos.

Para Estados Unidos, Leamer (1996) afirma que la ampliación de la brecha salarial entre trabajadores calificados y no calificados tiene, entre otras causas, la baja 
de precios en los bienes comerciables intensivos en mano de obra, producto de la globalización. Berman, E. et al. (1997) encuentran que esta creciente desigualdad está relacionada con el cambio tecnológico sesgado hacia el conocimiento, más que con el incremento comercial. Al extender su estudio a otros 10 países europeos de la OCDE, presentan evidencia de que en el periodo de 1970 a 1990 se produce una sustitución hacia el trabajo calificado en todos ellos, generando que sus salarios relativos se eleven o al menos permanezcan constantes.

Respecto a los países en desarrollo, las investigaciones empíricas son menos profusas y los resultados obtenidos van en las dos direcciones: algunos rechazando los teoremas de Heckher-Ohlin y Stolper-Samuelson, en el sentido de que el comercio internacional aumentó la demanda de trabajadores calificados y, por tanto, elevó la dispersión salarial en naciones menos desarrolladas; y otros aceptando las predicciones de la teoría convencional, al encontrar mejoramiento de la distribución salarial con la apertura externa.

En general, se ha encontrado que en los primeros países de menor desarrollo del sureste asiático, que iniciaron un proceso profundo de liberalización en las décadas de los sesenta y setenta, como Corea del Sur, Taiwán y Singapur, la demanda de trabajadores no calificados aumentó y la desigualdad salarial se redujo (Wood, 1994). Sin embargo, la experiencia de varias naciones en desarrollo de América Latina que han abierto su economía en las últimas dos décadas fue en el sentido contrario. Robbins (1994) analiza el periodo posterior a la apertura comercial en Chile $y$ encuentra que aumentó la demanda de trabajo calificado y su respectiva retribución con la liberalización; Galiani y Sanguinetti (2000) concluyen que la apertura comercial explica el crecimiento de la dispersión salarial en Argentina, aunque no tan fuerte como se esperaba; y Attanasio et al. (2003) encuentran que la política comercial influyó en la dispersión salarial por varias vías. Con datos del Censo Industrial Mexicano de 1965 a 1988, Hanson y Harrison (1999) observan que la ampliación de la brecha salarial estuvo asociada a la reforma comercial de 1985, sin grandes cambios en el empleo relativo. Empero, no hallan correlación significativa entre la intensidad del trabajo calificado y los cambios en los precios relativos al productor. De forma complementaria, Feestra y Hanson (1995) descubren que el incremento en la desigualdad salarial en México está asociado a los flujos de capital del exterior, particularmente de Estados Unidos, los cuales mueven su producción de bienes relativamente intensivos en trabajo calificado.

Investigadores de la primera etapa de la apertura (1985-1990), como Revenga (1995), asocian la reducción de tarifas a una disminución moderada del empleo y a 


\section{4}

BENJAMÍN BURGOS Y ALEJANDRO MUNGARAY

un incremento de los salarios promedio, particularmente de los trabajadores más calificados; Cragg y Epelbaum (1996) encuentran que la dispersión salarial crece poco explicada por los efectos específicos de la industria y en gran parte por los efectos específicos de la ocupación; y Feliciano (2001) descubre que la apertura comercial provocó un incremento de la desigualdad salarial al afectar industrias que tenían gran porcentaje de trabajadores no calificados.

\section{Metodología}

Para analizar el fenómeno de la desigualdad salarial en México y su relación con la apertura externa, se desarrollan dos enfoques, no relacionados entre sí. En primer lugar se utiliza la metodología simple del antes y después, de manera que se compara la situación previa a la apertura externa, en el año 1984, con la de los siguientes años hasta el momento actual. Bajo este primer enfoque se utilizan fuentes de datos en el nivel de personas a partir de dos estrategias. La primera es el cálculo y análisis de razones estadísticas e indicadores de desigualdad. La segunda de ellas emplea las funciones de ganancia desarrolladas por Mincer en 1974, en cuyo modelo estándar se corre la regresión del logaritmo de los salarios sobre un término constante, un término lineal de los años de escolaridad, términos lineal y cuadrático de los años de experiencia en el mercado de trabajo y otras características personales de los trabajadores. Los coeficientes correspondientes se interpretan como el retorno o premio por tener cada uno de esos atributos. En este estudio se estiman tales funciones mincerianas de retribuciones, haciéndose la regresión por mínimos cuadrados ordinarios del logaritmo de los salarios reales sobre algunas características personales de los trabajadores y otros indicadores relacionados con las actividades económicas y la apertura externa. Este enfoque ha sido utilizado con variantes por Green et al. (2000), Saba et al. (2004), Hanson (2003) y López-Acevedo (2003). En segundo lugar se avanza en ligar el proceso de apertura y sus diversos ritmos en los distintos subsectores de la industria manufacturera a lo largo del tiempo, con la desigualdad salarial. Con este enfoque se corren regresiones por el método de mínimos cuadrados generalizados factibles de indicadores de remuneración y empleo de trabajadores calificados y no calificados con variables relacionadas con el tamaño de la planta, el cambio tecnológico e indicadores de apertura externa, como tarifas arancelarias y exportaciones.

Las fuentes de datos utilizadas en el primer enfoque son las Encuestas Nacionales de Ingresos y Gastos de los Hogares (ENIGH), realizadas por el INEGI. Aquí se usan las referidas a 1984, año inmediatamente anterior al inicio de la apertura externa, 1989, 1992, 1994, 1996, 1998, 2000 y 2002, del periodo posterior. 
El tamaño de la muestra de las ENIGH, seleccionado aleatoriamente, ha crecido en el transcurso del tiempo. En 1984 estaban considerados 4,735 hogares y 23,985 personas en total, mientras que para 2002 dichas cifras eran 17,167 y 72,602 respectivamente. Dichas bases fueron depuradas para concentrarse en los asalariados de entre 16 y 65 años de edad que tuvieran empleo y que su ingreso trimestral fuera positivo. Se realizaron algunas depuraciones adicionales para solucionar incongruencias y evitar duplicidades en las bases de datos. Al final se trabajó con una base de datos que inició en 1984 con alrededor de 4,000 observaciones y terminó en 2002 con más de 15,000. En total, en el periodo analizado se incluyeron más de 81,000 observaciones.

Como indicador del salario se seleccionó el ingreso trimestral de los asalariados. Para convertirlo a precios constantes se utilizó el Índice Nacional de Precios al Consumidor con base en 2002, publicado por el Banco de México.

A partir de Green et al. (2000), los niveles educativos fueron clasificados en seis niveles:

1. ningún año de estudio; 2 . primaria incompleta; 3 . primaria completa y algunos años de secundaria; 4. secundaria completa y algunos años de bachillerato; 5 . bachillerato completo y algunos años de educación superior; 6 . licenciatura y posgrado completos.

La clasificación de los estados por regiones se realizó con el criterio de LópezAcevedo (2003), de tal manera que se definen cuatro regiones: la del sur, que en realidad incluye al sureste; la del norte, que no sólo incluye a los estados fronterizos, sino también los próximos a ellos; la del centro y la del Distrito Federal.

Por otra parte, la variable ocupación se seleccionó para ser utilizada como un indicador aproximado del tipo de trabajador, calificado y no calificado. De un lado se agruparon aquellas ocupaciones que requerirían de trabajadores más calificados (profesionistas, técnicos y personal especializado, funcionarios y directivos, etcétera); y por el otro aquellas que para desempeñarse no necesitarían de fuerza de trabajo calificada (trabajadores, operadores de maquinaria, ayudantes, peones, entre otros).

Por último, para el manejo de la información de las diversas actividades que desempeñaban los trabajadores, se utilizó la Clasificación Mexicana de Actividades Productivas (CMAP), que las divide en nueve sectores, además es en la que se basan las ENIGH anteriores. Sin embargo, como las ENIGH más recientes utilizan el Sistema de Clasificación Industrial de América del Norte (SCIAN), con mayor desglose, se realizaron las conversiones correspondientes para homogeneizarlas. 
Para observar los determinantes del salario real y precisar la evolución del pago a la escolaridad (calificación de la mano de obra), se utilizan ecuaciones de tipo minceriano y se hacen regresiones por el método de mínimos cuadrados ordinarios. En el cálculo de la matriz de varianzas y covarianzas, para tratar el problema de la heteroscedasticidad, se hace uso del método de White, el cual parte de estimar y emplear una matriz de varianzas y covarianzas del término del error consistente con heteroscedasticidad. La variable dependiente es el salario real a precios constantes de 2002, y como variable independiente se tienen variables dummy, en su mayoría relacionadas con la escolaridad, región, sectores económicos y otros aspectos.

Entre las variables independientes empleadas están la edad y la edad al cuadrado; una variable dummy para el género (la categoría excluida es la mujer); una para la zona (para las ecuaciones de 1984 y 1989 la categoría excluida es la de baja densidad, mientras que para el resto de años se abren tres o cuatro variables dummy según los estratos utilizados); variables dummy para cinco niveles de escolaridad (la categoría excluida es el nivel 1, que se refiere a los que no tienen aprobado ningún grado de educación formal); variables dummy para tres regiones (la región excluida es el sur); una variable dummy para el tipo de empleo según se requiera de mayor calificación o no (la categoría excluida se refiere a las ocupaciones que no requieren de mayor calificación); una variable dummy para reflejar si el trabajador está sindicalizado o no (este último caso se excluye). De esta forma, la especificación econométrica del modelo quedaría como sigue:

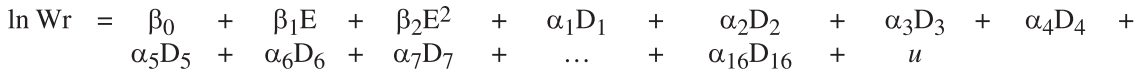

Donde $W r$ son los salarios reales y $E$ es la edad. Las $D$ son las variables dummy que permiten incluir los aspectos cualitativos: $D_{1}, D_{2}, D_{3}$ y $D_{4}$ para los cinco estratos poblacionales; $D_{5}$ para el sexo; $D_{6}, D_{7}, D_{8}, D_{9}$ y $D_{10}$ para los seis niveles educativos; $D_{11}, D_{12}$ y $D_{13}$ para las cuatro regiones; $D_{14}$ para el tipo de empleo; $D_{15}$ para la pertenencia a sindicato; y $D_{16}$ para el tipo de bienes (comerciables y no comerciables). Al utilizar el software econométrico EViews 5.0, se corren las regresiones por el método de mínimos cuadrados ordinarios para estimar los parámetros poblacionales $(\alpha, \beta)$ a partir de reducir los errores al cuadrado.

Por otra parte, para lograr una correlación más directa de la apertura externa con los cambios en los precios relativos, las modificaciones en la demanda relativa de empleo calificado y no calificado y con las consecuentes modificaciones en la retribución relativa a ambos tipos de trabajadores, se ha recurrido a información de empresas en los Censos Económicos que se realizan periódicamente, pues contienen datos sobre los 
activos, la formación bruta de capital, el valor agregado y otros que permiten tipificar a las compañías e industrias. En este trabajo se utilizan los correspondientes al periodo analizado: 1985, 1988, 1993 y 1998. El análisis se realiza centrándose en el sector manufacturero en el nivel industria de dos dígitos (subsector) y de cuatro dígitos (ramas).

Todas las variables expresadas en términos monetarios, como salarios, activos fijos totales, valor agregado censal bruto, formación bruta de capital, fueron deflactadas con base en el Índice Nacional de Precios al Consumidor de la segunda quincena de junio de 2002. Desafortunadamente las fuentes de datos en el nivel de planta, como es el caso de los Censos Económicos, no contienen suficiente información para realizar una distinción precisa del trabajo calificado y no calificado.

Así, a partir de la variable ocupación de las ENIGH, la variable indicadora del pago relativo del trabajo calificado y no calificado se obtuvo con la razón del salario medio anual por empleado entre el salario medio anual del obrero, y para analizar los cambios en el empleo relativo se obtuvo la razón promedio de trabajadores ocupados entre el promedio de obreros ocupados. El indicador de cambios en los precios relativos se logró dividiendo el Índice de Precios al Productor de cada una de las industrias, entre el correspondiente al de toda la industria manufacturera.

Por la naturaleza de los datos utilizados de los Censos Económicos, con información de las diversas industrias en cuatro puntos en el tiempo, se eligieron métodos de regresión que aplican a datos de panel, con los 36 datos del periodo. Este tipo de referencias son observaciones sobre las mismas unidades transversales a lo largo de varios intervalos de tiempo, y el procedimiento de estimación varía en función de los supuestos con respecto a los coeficientes de la intersección y de las pendientes, así como sobre el término del error.

Como variables dependientes se utilizó la razón de los salarios de trabajadores calificados respecto a los salarios de los no calificados (rseo) y la proporción de empleo calificado (resu). Como variables explicatorias se usaron dos grupos: el primero de ellos conteniendo aquellas relacionadas con las medidas y resultados de la apertura externa, tales como tarifas arancelarias (tar), monto de exportaciones (exp) y precios relativos $(p r)$; y el segundo grupo integrado por variables que reflejan la evolución y cambios de los procesos de producción, tales como activos, activos por persona ocupada, formación bruta de capital, valor agregado, entre otras. Todas ellas se expresan en su forma logarítmica para trabajar con las respectivas tasas de crecimiento, con lo cual la especificación econométrica quedaría como sigue:

$$
\ln \text { rseo }=\beta_{0}+\beta_{1} \ln \operatorname{tar}+\beta_{2} \ln \exp +\beta_{3} \ln \mathrm{pr}+\sum_{i=4} \beta_{i} \ln X_{i}+\mathrm{u}
$$


Donde la $X_{i}$ representa el conjunto de variables del segundo grupo (activos, formación bruta de capital, valor agregado, etcétera) y $u$ el término del error. En el caso de la segunda regresión sólo se sustituye ln rseo por ln resu.

En un primer momento se utilizaron los modelos de efectos aleatorios y fijos. Para la selección de alguno de estos dos métodos se aplicó la prueba de Hausman, la cual parte de la hipótesis nula de que los estimadores obtenidos a partir de ambos métodos difieren sustancialmente y usa el estadístico de prueba $X^{2}$. Como resultado se rechazó dicha hipótesis y se concluyó que era conveniente utilizar el segundo método. Sin embargo, en las diversas regresiones se encontró que existía heteroscedasticidad a lo largo de los páneles (entre las diversas industrias) y autocorrelación dentro de los páneles. Esto se resolvió con el método de mínimos cuadrados generalizados factibles, que permite la estimación con la presencia de ambos problemas, el cual consiste en correr, en una primera instancia, la regresión por mínimos cuadrados ordinarios, obtener los residuales, formar la matriz de varianzas y covarianzas y volver a correr la regresión incorporando estos resultados. Estas regresiones se realizaron con el software Stata 8.

De acuerdo con los datos de las ENIGH, durante el periodo de la apertura externa se ha observado una tendencia hacia la dispersión. Cualesquiera de las medidas seleccionadas de desigualdad salarial que se incluyen en el cuadro 1 muestran un incremento constante en la dispersión salarial a lo largo del periodo analizado, con excepción de los años posteriores a la crisis de 1995 y de desaceleración del crecimiento de 2000 en adelante.

Cuadro 1

Medidas de dispersión salarial e índices de desigualdad

\begin{tabular}{cccc}
\hline Año & $\begin{array}{c}\text { Coeficiente } \\
\text { de Gini }\end{array}$ & Índice de Theil & $\begin{array}{c}\text { Desviación logarítmica } \\
\text { media }\end{array}$ \\
\hline 1984 & 0.3809 & 0.2638 & 0.3037 \\
1989 & 0.4199 & 0.3276 & 0.3411 \\
1992 & 0.46849 & 0.418117 & 0.42562 \\
1994 & 0.49748 & 0.50056 & 0.47964 \\
1996 & 0.4951 & 0.4632 & 0.4827 \\
1998 & 0.50435 & 0.48805 & 0.5044 \\
2000 & 0.4683 & 0.4202 & 0.4142 \\
2002 & 0.4493 & 0.3757 & 0.3866 \\
\hline
\end{tabular}

Por otra parte, los datos de las ENIGH muestran cambios en los niveles de escolaridad de los asalariados empleados en los diversos sectores productivos del país. El 
cuadro 2 muestra claramente que la demanda de trabajadores con poca calificación (niveles 1, 2 y 3 ) ha bajado a lo largo del periodo analizado, mientras que la de los mayores niveles de escolaridad $(4,5$ y 6$)$ muestra una tendencia al crecimiento.

Cuadro 2

Porcentaje de trabajadores según nivel educativo

\begin{tabular}{ccccccc}
\hline Año & $\begin{array}{c}\text { Nivel 1 } \\
(\%)\end{array}$ & $\begin{array}{c}\text { Nivel 2 } \\
(\%)\end{array}$ & $\begin{array}{c}\text { Nivel 3 } \\
(\%)\end{array}$ & $\begin{array}{c}\text { Nivel 4 } \\
(\%)\end{array}$ & $\begin{array}{c}\text { Nivel 5 } \\
(\%)\end{array}$ & $\begin{array}{c}\text { Nivel 6 } \\
(\%)\end{array}$ \\
\hline 1984 & 9.86 & 25.33 & 29.14 & 18.62 & 10.41 & 6.64 \\
1989 & 8.09 & 18.11 & 27.23 & 24.70 & 13.92 & 7.95 \\
1992 & 7.00 & 18.64 & 27.11 & 27.33 & 12.50 & 7.43 \\
1994 & 7.32 & 17.60 & 25.12 & 27.30 & 13.60 & 9.06 \\
1996 & 5.24 & 15.71 & 24.51 & 30.24 & 15.32 & 8.99 \\
1998 & 5.68 & 14.78 & 25.42 & 30.05 & 14.97 & 9.10 \\
2000 & 4.19 & 13.27 & 23.70 & 31.67 & 15.27 & 11.90 \\
2002 & 4.60 & 13.08 & 22.00 & 32.09 & 18.48 & 9.74 \\
\hline
\end{tabular}

Al realizar la clasificación de todos los empleados en función de la variable ocupación se encontró que la proporción de trabajadores calificados respecto a los no calificados no presentó cambio sustancial en el periodo. De hecho, en los últimos 10 años permaneció prácticamente sin variaciones mayores, siendo alrededor de 78 el porcentaje de trabajadores no calificados y $22 \%$ los calificados. Cabe aclarar que la discrepancia de estos resultados con los del cuadro anterior podría explicarse por la no correspondencia exacta del tipo de ocupación con el nivel de escolaridad de los trabajadores.

En cambio, el aumento en la razón de los salarios reales pagados a los trabajadores calificados respecto al de los no calificados sí es muy evidente, como se observa en el cuadro 3 .

Cuadro 3

Salarios reales por tipo de empleo

\begin{tabular}{ccccc}
\hline Año & $\begin{array}{c}\text { Promedio } \\
\text { total }\end{array}$ & $\begin{array}{c}\text { Promedio } \\
\text { empleo no } \\
\text { calificado }\end{array}$ & $\begin{array}{c}\text { Promedio } \\
\text { empleo } \\
\text { calificado }\end{array}$ & $\begin{array}{c}\text { Razón } \\
\text { salarios } \\
\text { cal./no cal. }\end{array}$ \\
\hline 1984 & 10,476 & 8,036 & 16,113 & 2.01 \\
1989 & 10,768 & 8,234 & 16,220 & 1.97 \\
1992 & 12,714 & 9,641 & 26,636 & 2.76 \\
1994 & 14,058 & 9,502 & 31,316 & 3.30 \\
1996 & 9,402 & 7,135 & 19,011 & 2.66 \\
1998 & 9,950 & 7,600 & 20,059 & 2.64 \\
2000 & 12,218 & 8,572 & 24,276 & 2.83 \\
2002 & 12,260 & 8,894 & 25,135 & 2.83 \\
\hline
\end{tabular}


Respecto a la retribución relativa según los distintos niveles de escolaridad definidos, de acuerdo con la regresión de las ecuaciones del cuadro 4, como era de esperarse los niveles salariales muestran una relación directa con el nivel educativo. En todos los años, entre mayor es el nivel educativo del trabajador mayor es su remuneración real. En 1984 los trabajadores con grado de licenciatura y posgrado percibían salarios [(exp(1.103887)-1)*100], 201.6\% más que los de menor calificación y [exp(1. 103817-0.801803)-1)*100], 35.3\% más elevados que los que habían terminado sus estudios de preparatoria o tenían educación superior incompleta. Para 2002, dichos porcentajes fueron de $319.5 \%$ y $45.2 \%$, respectivamente.

Cuadro 4

Resumen de los resultados de las regresiones por MCO del logaritmo de los salarios reales

\begin{tabular}{|c|c|c|c|c|c|c|c|c|}
\hline \multirow{2}{*}{ Variable } & \multirow[b]{2}{*}{1984} & \multirow[b]{2}{*}{1989} & \multicolumn{6}{|c|}{ Años } \\
\hline & & & 1992 & 1994 & 1996 & 1998 & 2000 & 2002 \\
\hline Edad & $0.0797679^{*}$ & $0.0725603^{*}$ & $0.0499715^{*}$ & $0.0588006^{*}$ & $0.0492563^{*}$ & $0.0690988^{*}$ & $0.046695^{*}$ & $0.036449^{\prime}$ \\
\hline Edad al cuadrado & $-0.0009263^{*}$ & $-0.0008677^{*}$ & $-0.0005565^{*}$ & $-0.0006443^{*}$ & $-0.0005747^{*}$ & $-0.0007984^{*}$ & $-0.0005806^{*}$ & -0.0004209 \\
\hline Estrato 2 & $0.3822005^{*}$ & $0.3198775^{*}$ & $0.305884^{*}$ & $0.2376101^{*}$ & $0.1918596^{*}$ & $0.2987418^{*}$ & $0.1998998 *$ & $0.1637666^{\circ}$ \\
\hline Estrato 3 & & & $0.4173309^{*}$ & $0.2981388^{*}$ & $0.2951806^{*}$ & $0.2830388^{*}$ & $0.3640067^{*}$ & $0.2546924^{\prime}$ \\
\hline Estrato 4 & & & $0.5629824^{*}$ & $0.4773253^{*}$ & $0.473256^{*}$ & $0.4352638 *$ & $0.4953644^{*}$ & $0.4422426^{\prime}$ \\
\hline Estrato 5 & & & $0.5399175^{*}$ & $0.4573756^{*}$ & $0.4383286^{*}$ & $0.5676832^{*}$ & & \\
\hline Sexo (Hombre) & $0.4235296^{*}$ & $0.1742919^{*}$ & $0.173358 *$ & $0.1735197^{*}$ & $0.1102771^{*}$ & $0.1905964^{*}$ & $0.069282^{*}$ & 0.12313 \\
\hline tivo 2 & $0.4582463^{*}$ & $0.1758284^{*}$ & $0.1758343^{*}$ & $0.1599031^{*}$ & $0.0750583^{*}$ & $0.1621159^{*}$ & $0.3003164^{*}$ & $0.2130113^{3}$ \\
\hline livo 3 & $0.7367779 *$ & $0.392327^{*}$ & $0.3873648^{*}$ & $0.4024514^{*}$ & $0.4037375^{*}$ & $0.369702^{*}$ & $0.4873345^{*}$ & $0.3554114^{4}$ \\
\hline Nivel educativo 4 & $0.7994033^{*}$ & $0.5249142^{*}$ & $0.587844^{*}$ & $0.6280549^{*}$ & $0.5927295^{*}$ & $0.5564953^{*}$ & $0.6143933^{*}$ & $0.5391223^{3}$ \\
\hline Nivel educativo 5 & $0.8018028 *$ & $0.6968182^{*}$ & $0.7625746^{*}$ & $0.9312118^{*}$ & $0.8622405^{*}$ & $0.8268516^{*}$ & $0.8220258 *$ & 0.7887819 \\
\hline ivo 6 & $1.103817^{*}$ & $1.028583^{*}$ & $1.103311^{*}$ & 1.332729* & $1.201264^{*}$ & $1.214203^{*}$ & $1.241095^{*}$ & $1.161663^{\circ}$ \\
\hline Regiones (Centro) & $0.0207526^{*}$ & $0.1475225^{*}$ & $0.1123359^{*}$ & $0.2091205^{*}$ & $0.0174604^{*}$ & $0.0516299 *$ & $0.2393701^{*}$ & $0.2774294^{\prime}$ \\
\hline Regiones (Norte) & $0.1142511^{*}$ & $0.1745957^{*}$ & $0.1479976^{*}$ & $0.1769634^{*}$ & $0.1977049^{*}$ & $0.2524081^{*}$ & $0.3663845^{*}$ & $0.3945916^{\prime}$ \\
\hline Regiones (DF) & $0.1836412^{*}$ & $0.1024153^{*}$ & 0.2098066 * & $0.2894968^{*}$ & $0.2101977^{*}$ & $0.154209^{*}$ & $0.3245191^{*}$ & $0.3152442^{\prime}$ \\
\hline $\begin{array}{l}\text { Tipo de empleo } \\
\text { (calificado) }\end{array}$ & $0.4114666^{*}$ & $0.2383617^{*}$ & $0.3924444^{*}$ & $0.3623356^{*}$ & $0.345851^{*}$ & $0.298104^{*}$ & $0.4006866^{*}$ & $0.3729689^{\circ}$ \\
\hline Sindicalizado & $0.2461399^{*}$ & $0.0784251^{*}$ & $0.1075104^{*}$ & $0.0552738^{*}$ & $0.1761577^{*}$ & $0.2134016^{*}$ & $0.0865535^{*}$ & $0.209115^{\circ}$ \\
\hline & $-0.0123282^{*}$ & $-0.0874734^{*}$ & $-0.0332847^{*}$ & $-0.0910811^{*}$ & $-0.0146476^{*}$ & $0.0570342^{*}$ & $0.0008718^{* *}$ & $-0.0550144^{\prime}$ \\
\hline Constante & 5.948909* & $6.638357^{*}$ & $6.895463^{*}$ & $6.703695^{*}$ & $6.706057^{*}$ & $6.174978^{*}$ & $6.762708^{*}$ & 7.025877 \\
\hline
\end{tabular}

* Significativo a $1 \%$

** Significativo a $5 \%$

Por otro lado, puede apreciarse una tendencia general de decremento de la remuneración relativa en los niveles salariales bajos y un crecimiento en los niveles altos 
(véase cuadro 5). El aumento de la dispersión salarial también puede apreciarse cuando los puntos log entre los niveles en 1984 no eran tan grandes, pues la diferencia entre el nivel 2 y el 6 era de 6.5, mientras que entre los niveles 3,4 , y 5 era muy pequeña (menor a 1 punto log). Para 1989 esa situación cambia considerablemente, pues la diferencia entre ambos extremos aumenta a 8.5 puntos log y la distancia entre los niveles intermedios se ubica entre 1 y 3 puntos log. El punto de mayor dispersión salarial se ubica en 1994, cuando la distancia entre los niveles educativos 2 y 6 se ubica en 11.7 puntos log, mientras que los niveles intermedios se separan por entre 2 y 5 puntos log. Para 2002 se observan también amplios diferenciales en la retribución salarial de los trabajadores de acuerdo con su nivel educativo: entre los niveles 2 y 6 se encuentran con 9.5 puntos $\log$ de diferencia y entre los niveles 3 y 6 existen más de 8 puntos log.

Cuadro 5

Evolución de la retribución relativa a la escolaridad

\begin{tabular}{llllll}
\hline \multirow{2}{*}{ Año } & \multicolumn{5}{c}{ Niveles } \\
\cline { 2 - 6 } & Nivel 2 & Nivel 3 & Nivel 4 & Nivel 5 & Nivel 6 \\
\hline 1984 & 0.458246 & 0.736778 & 0.799403 & 0.801803 & 1.103817 \\
1989 & 0.175828 & 0.392327 & 0.524914 & 0.696818 & 1.028583 \\
1992 & 0.175834 & 0.387365 & 0.587844 & 0.762575 & 1.103311 \\
1994 & 0.159903 & 0.402451 & 0.628055 & 0.931212 & 1.332729 \\
1996 & 0.075058 & 0.403738 & 0.592730 & 0.862241 & 1.201264 \\
1998 & 0.162116 & 0.369702 & 0.556495 & 0.826852 & 1.214203 \\
2000 & 0.300316 & 0.487335 & 0.614393 & 0.822026 & 1.241095 \\
2002 & 0.213011 & 0.355411 & 0.539122 & 0.788782 & 1.161663 \\
\hline
\end{tabular}

En general, puede afirmarse que después de 1984, año previo a la apertura externa en México, las brechas salariales de los trabajadores según su distinto nivel educativo tendieron a ensancharse. Por su amplitud destacan las correspondientes a los dos niveles de mayor escolaridad. Por otra parte, los niveles 3 y 4 , que iniciaron con un premio similar al que tenía el nivel 5 , fueron los que mayores cambios observaron, al separarse durante el periodo analizado entre 3 y 5 puntos log del nivel 5 .

Se puede destacar que si bien se observa un proceso claro de aumento de la desigualdad salarial en función de los distintos niveles de escolaridad, éste no ha sido lineal, pues ha habido años en que se detuvo o se retrocedió temporalmente en esa tendencia a pesar del avance de la apertura comercial. Como factores explicativos de dicha situación se pueden señalar el incremento de la oferta de trabajo — en parte por la incorpo- 
ración de la mujer al mercado laboral—, los problemas económicos del país durante el periodo analizado (la crisis de 1995 y la desaceleración económica observada a partir del año 2001) y los aumentos en la población con mayores niveles de escolaridad, particularmente por el crecimiento de la educación universitaria. Si bien ésta inició en la década de los setenta, se aceleró a mediados de los noventa.

Como era de esperarse, el incremento de la matrícula de licenciatura en esos años se reflejó en un aumento importante en el número de egresados de los primeros años de la presente década: de 103,280 en 1985 a 249,085 en 2002 (ANUIES, Anuario Estadístico 2003). Estos datos pueden explicar el retroceso en la remuneración relativa de los trabajadores calificados observada en 2002.

A partir de la ocupación reportada en las ENIGH, los resultados de la regresión indican que el valor estimado resultó significativo en todos los años y reflejan que existe un premio salarial a las ocupaciones que requieren mayor nivel de calificación, el cual se ubica en 0.238 en 1987, alcanza su máximo en 2000 con 0.400 y termina en 0.372 en 2002, sin considerar el año 1984. De nueva cuenta se observa una tendencia a aumentar la remuneración de los trabajadores más calificados.

La edad como indicador de la experiencia potencial resultó significativa en todos los años analizados, habiéndose encontrado que también existe un premio salarial por año de edad, el cual disminuyó a lo largo del periodo analizado. El pico de las remuneraciones según la edad se ubica en 43.1 años en 1984 y 43.3 en 2002.

De acuerdo con los resultados esperados, el género también influye en los salarios reales de los trabajadores, ya que se identifican mayores remuneraciones de los hombres que las mujeres. En el periodo analizado, el diferencial amplio en 1984 tendió a reducirse hasta niveles de $12 \%$ en 2002. Los resultados muestran un premio salarial a la sindicalización, el cual es variable en el periodo analizado, comenzando con 0.2461 en 1984 y terminando en 0.2091 en 2002.

El tamaño de la localidad también resultó un factor que influye en los salarios reales de los trabajadores. Como era de esperarse, a menor densidad poblacional de la localidad, menor salario real en todos los años de los que se disponen datos. Sin embargo, es necesario destacar que los diferentes estratos de población a lo largo del periodo analizado redujeron su premio salarial respecto a las localidades de menor población. Entre los niveles también se observa esa disminución.

En cuanto a las diversas regiones definidas, se encontró que existen diferencias salariales relacionadas con la región en la que viven los trabajadores. La región sur es la de menores percepciones salariales y la del Distrito Federal la de mayores. En el periodo de estudio se observó una tendencia a aumentar las remuneraciones relativas 
en todas las regiones en relación con la región sur, especialmente la norte con un mayor crecimiento en el premio salarial, que inició con 0.1142 en 1984 y terminó con 0.3945 en 2002, nivel superior al de la zona del Distrito Federal.

En regresiones de la misma ecuación, pero por sector productivo, se obtuvieron resultados no homogéneos. Por un lado, en varios casos los coeficientes no alcanzaron la significancia requerida. En algunos sectores como la manufactura, el comercio y los servicios, se observa más claramente el aumento de la dispersión salarial en el periodo analizado.

Los resultados obtenidos hasta aquí, bajo este primer enfoque con datos fundamentalmente sobre personas obtenidos de las ENIGH, permiten observar la tendencia a aumentar la desigualdad salarial durante el proceso de apertura comercial en México. Para avanzar en mostrar de manera directa la relación entre ambas, se utiliza el segundo enfoque.

La evolución observada en las distintas variables posibilita obtener algunas conclusiones iniciales sobre tal relación. Con el uso de la variable valor agregado como indicador del nivel de actividad de cada una de las industrias, se puede apreciar que en general todas crecieron entre 1985 y 1998 a tasas entre $9 \%$ y 105\%, siendo las de mayor crecimiento la de maquinaria y equipo, otras industrias, papel y alimentos (las menos intensivas en capital), mientras que las de menor crecimiento son las metálicas y química, más intensivas en capital.

Gráfica 1

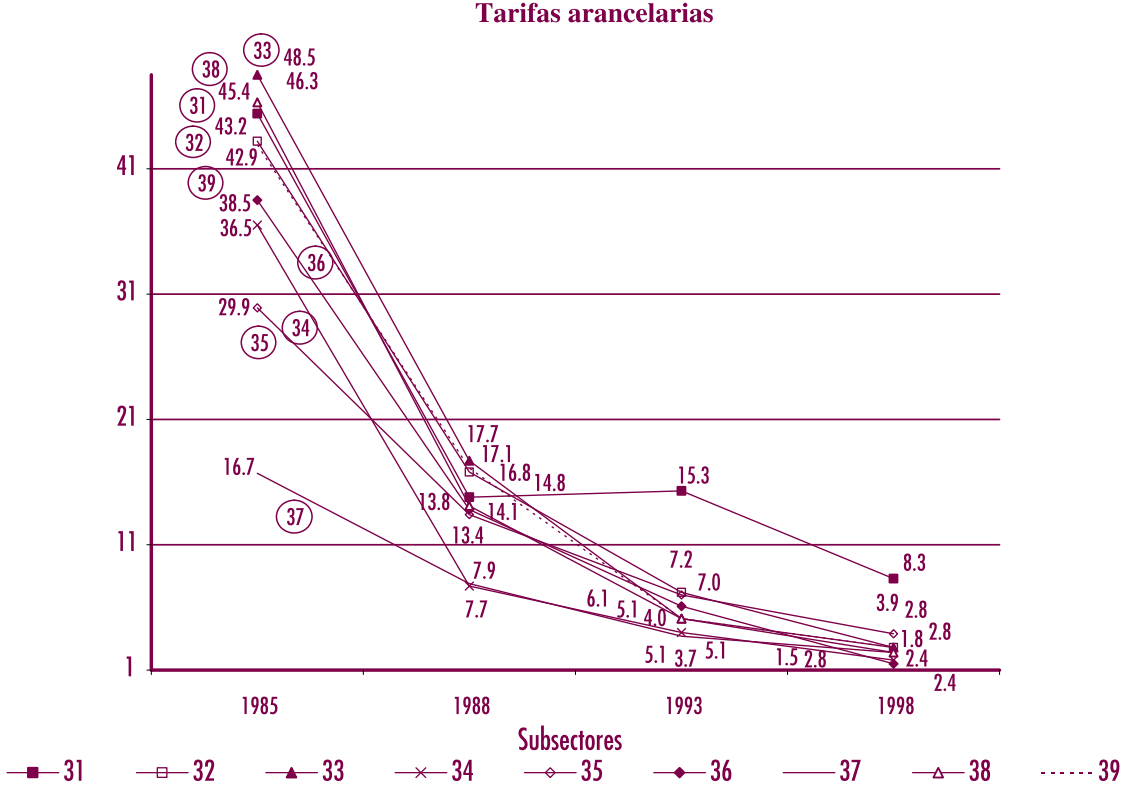


Respecto a las tarifas a la importación de bienes de cada una de las industrias, se puede apreciar que inicialmente existía una dispersión de tarifas por industrias, la cual tendió a reducirse con el paso del tiempo. También puede observarse que, en general, las diversas industrias siguieron un mismo ritmo en cuanto a la reducción de tarifas, con excepción de las del papel, que al principio caen mucho más rápido que las demás y la alimenticia, que inicialmente disminuye al mismo nivel que el resto y posteriormente baja su ritmo de reducción.

En 1985 las industrias con tarifas más altas eran las de productos de madera, maquinaria y equipo, alimentos y textiles, que constituían el grupo de las de menor intensidad de capital en ese año; mientras que las de menores tarifas eran las metálicas, las químicas y papel y vidrio, que coincidentemente eran las de mayor intensidad de capital.

Frecuentemente se cita la liberalización comercial como un factor que incide en la corrección del crecimiento diferenciado de los precios en las distintas industrias. Su evolución en el periodo analizado está más ligada a la apertura externa, según se puede apreciar en la gráfica 2.

Gráfica 2

Precios relativos

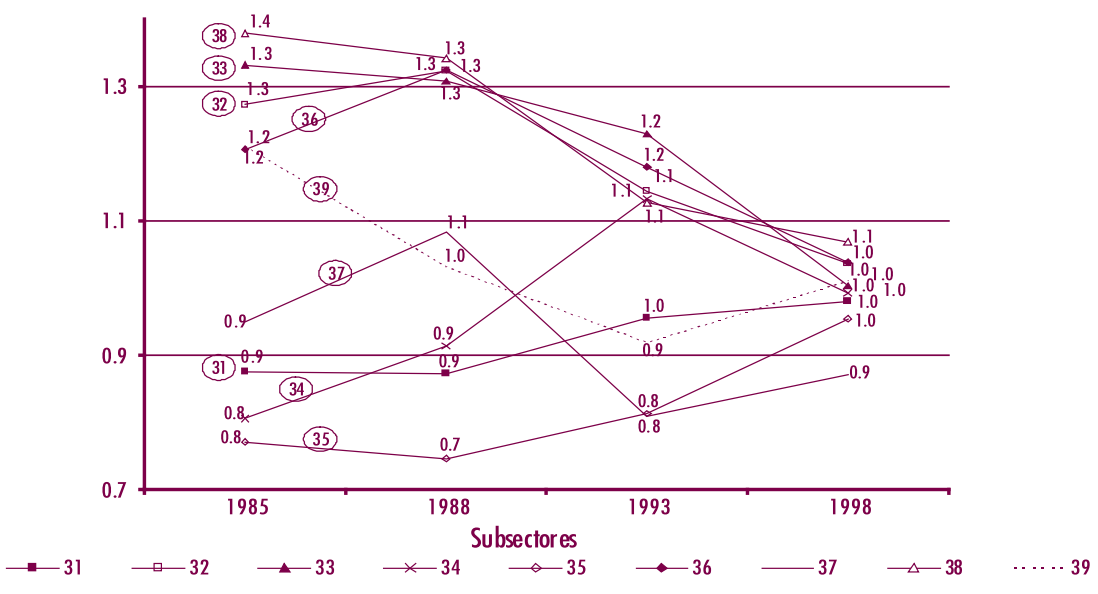

En primer lugar se observa un crecimiento dispar de los precios relativos entre las diferentes industrias al iniciar la liberalización comercial. Asimismo, se advierte que con el paso del tiempo desde ambos extremos se tiende a que los precios tengan un crecimiento uniforme. También es destacable que las industrias con precios relativos 
mayores al inicio fueron precisamente las que tenían mayores niveles de protección antes de la apertura. Tal es el caso de las industrias de maquinaria y equipo, productos de madera, textiles y vidrio, mientras que las de menores precios relativos corresponden a las industrias de química, papel y alimentos que, con excepción de esta última, eran las de menor importancia.

Gráfica 3

Exportaciones

(millones de dólares)

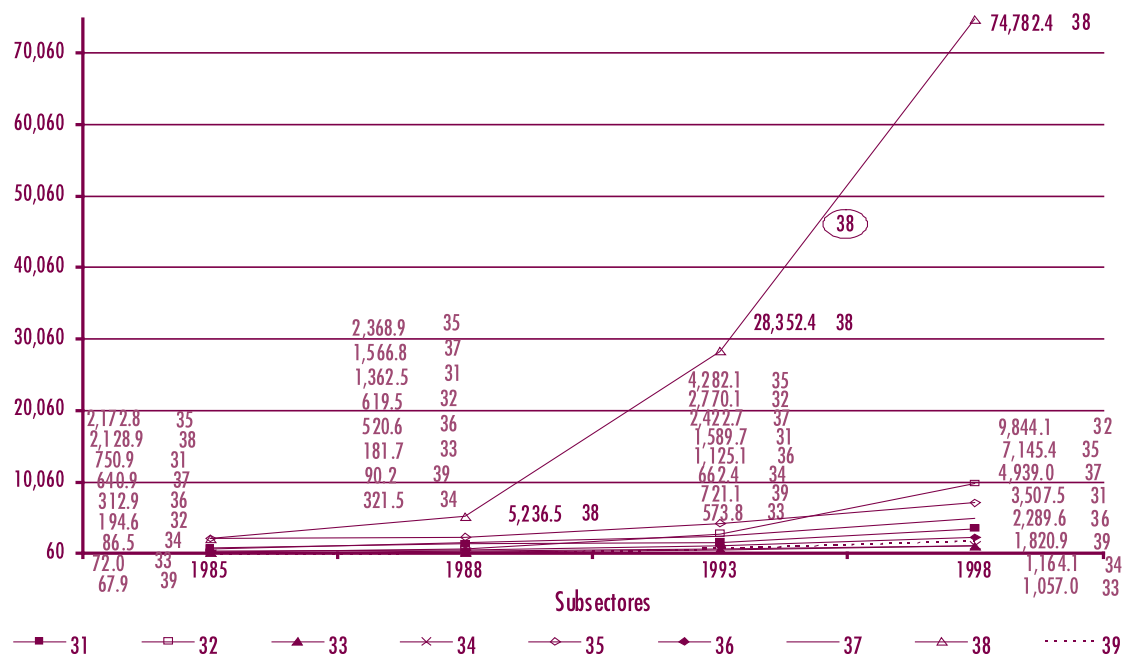

En general, las exportaciones de la industria manufacturera mostraron un crecimiento en el periodo analizado. En la gráfica 3 destaca el gran crecimiento de las exportaciones del subsector de maquinaria y equipo, seguido a una distancia considerable por las industrias de textiles, química y metálicas.

El indicador construido para medir la evolución de los salarios relativos de la mano de obra calificada respecto de la no calificada muestra la misma tendencia que los indicadores utilizados en las otras bases de datos. El comportamiento de la razón de salarios pagados a los empleados en relación con el pagado a los obreros indica que en todas las industrias existe una tendencia al crecimiento de la desigualdad salarial. Sólo al final se revierte la de la industria de productos de madera.

En general, las diversas industrias conservan la posición relativa que tenían al inicio en 1985. Las que cambiaron fueron las industrias química, metálica y de vidrio 
con tendencia al alza, y la alimenticia y textiles a la baja. Las primeras, más intensivas de capital y las segundas de menor intensidad. Esto es compatible con la hipótesis de que el capital y el trabajo calificado son complementarios y que, por tanto, tienda a crecer la remuneración relativa de ese tipo de mano de obra.

Gráfica 4

Razón salarios de empleados calificados /no calificados

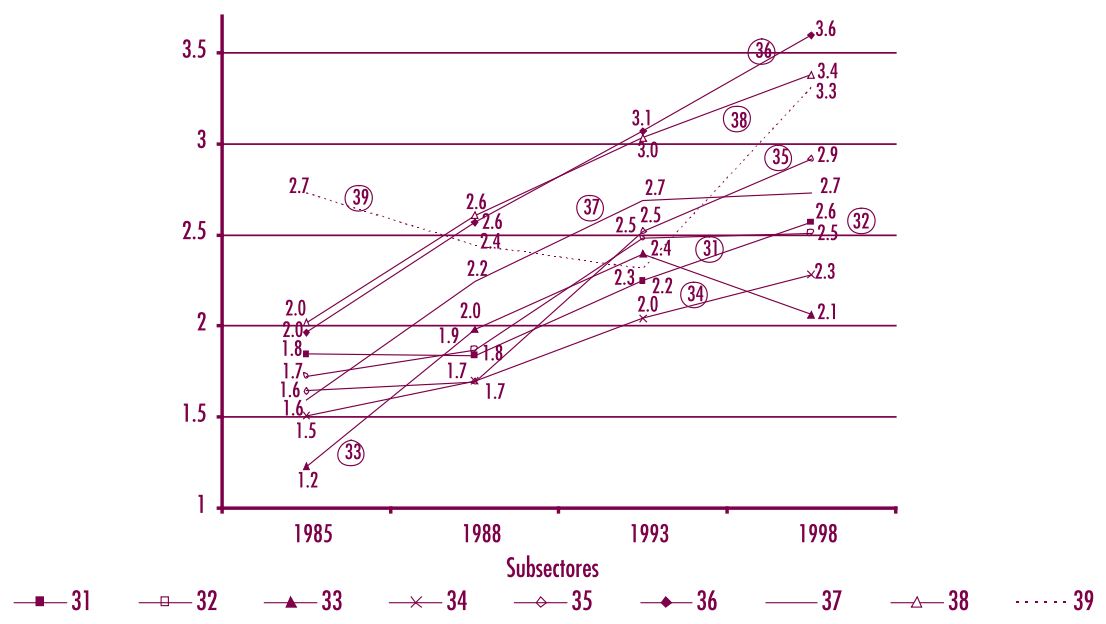

Parte del mecanismo de transmisión de los efectos de la liberalización comercial sobre la remuneración relativa al personal, según el teorema de Stolper-Samuelson, son los cambios en la demanda relativa de trabajo calificado en relación con la del no calificado.

En el periodo analizado no se presenta un cambio sustancial en dicho indicador, ni una tendencia definida para el conjunto de las industrias. Algunas de ellas terminan con una ligera alza (química, metálica y alimentos) y otras con una ligera baja (textiles, productos de madera, maquinaria y equipo), lo cual es en general compatible con la naturaleza de la intensidad del capital, alta en las primeras y baja en las segundas. Es posible que la no congruencia de estos resultados con los mostrados en el cuadro 2 sea explicada no sólo porque en aquel caso se utilizan datos del conjunto de la economía y aquí únicamente del sector manufacturero, sino particularmente por la no correspondencia precisa del nivel de escolaridad de los trabajadores con el tipo de empleo (calificado o no calificado) efectivamente desarrollado. 
Gráfica 5

Razón empleo calificado/no calificado

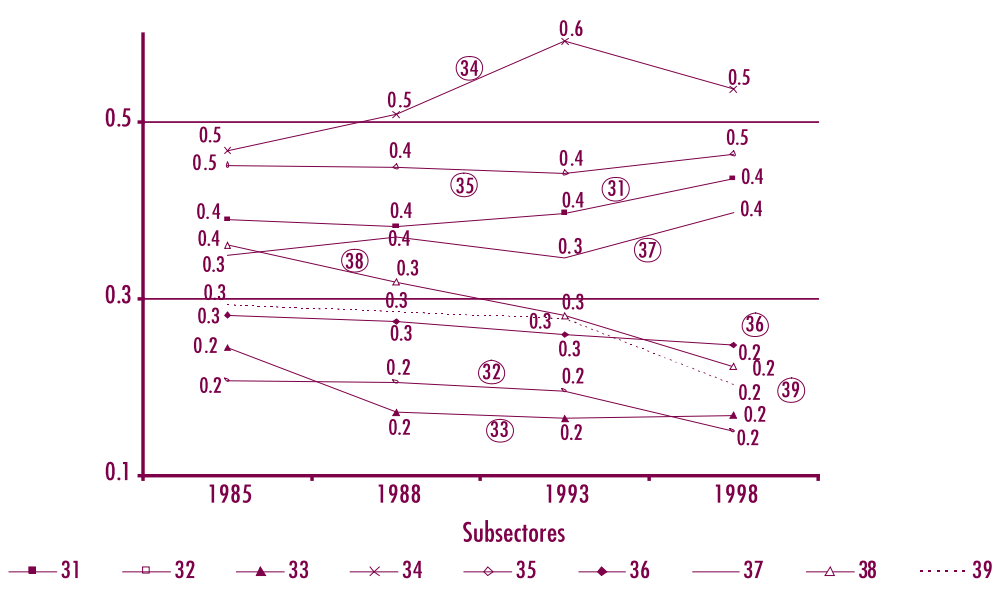

Con los datos de las industrias en el nivel de dos dígitos ninguna variable del primer grupo resultó significativa para explicar el crecimiento observado en la razón de los salarios de la mano de obra calificada, en relación con los de la mano de obra no calificada ( $r s e o$ ). Por tanto, para el caso mexicano la tendencia observada a la desigualdad salarial no puede explicarse mediante los cambios en la reorganización de los procesos productivos en las diversas industrias. Sin embargo, se encontró un pequeño efecto entre los cambios en las tarifas arancelarias (tar) y los cambios en los precios relativos (pr) como mecanismo de transmisión original (véase cuadro 6). Ello indicaría que la baja en los precios relativos observada en la mayoría de las industrias estuvo relacionada con la baja en las tarifas arancelarias que se manifestó durante el periodo.

\section{Cuadro 6}

\begin{tabular}{lcccccc}
\hline Ipr & Coeficientes & Error estándar & $z$ & $P>|z|$ & [95\% intervalo de confianza] \\
\hline Itar & 0.0470724 & 0.0205564 & 2.29 & 0.022 & 0.0067826 & 0.0873622 \\
cons & -0.0464629 & 0.0571971 & -0.81 & 0.417 & -0.158567 & 0.0656413 \\
\hline
\end{tabular}

Wald chi2 $(1)=5.24 ;$ Prob $>$ chi $2=0.0220$

Además de los precios relativos, es notorio que las variables relacionadas con la apertura externa sí resultaron significativas para explicar el crecimiento en la desigualdad salarial (véase cuadro 7). 
Cuadro 7

\begin{tabular}{lcccccc}
\hline Irseo & Coeficientes & Error estándar & $z$ & $P>|z|$ & [95\% intervalo de confianza] \\
\hline Itar & -0.13784462 & 0.0239215 & -5.76 & 0.000 & -0.1847314 & -0.090961 \\
Ivar 26 & 0.0546191 & 0.0148407 & 3.68 & 0.000 & 0.0255318 & 0.0837063 \\
Ipr & 0.3918741 & 0.1168402 & 3.35 & 0.001 & 0.1628714 & 0.6208768 \\
cons & 0.7096644 & 0.168825 & 4.20 & 0.000 & 0.3787734 & 1.040555 \\
\hline
\end{tabular}

Wald chi2 $(3)=190.44 ;$ Prob $>$ chi $2=0.0000$

Como puede apreciarse, los coeficientes obtenidos y sus signos son compatibles con el resto de resultados obtenidos en este trabajo. La baja en las tarifas elevó la razón de salarios de trabajadores calificados/no calificados, al igual que el aumento en las exportaciones (exp). La apertura externa tuvo el efecto de acrecentar la desigualdad salarial, contrario a lo esperado por la teoría convencional para un país en desarrollo, la cual predecía que con la liberalización comercial aumentarían la demanda, la producción y el precio de los bienes intensivos en mano de obra no calificada, por ser el factor abundante en el país, así como el salario de tales trabajadores, disminuyendo con ello la desigualdad salarial.

Existen varias explicaciones posibles a esos resultados, cuyos elementos ya han sido tratados en este trabajo. En primer lugar, en la línea de argumentación de Davis, seguramente México tiene menor abundancia de mano de obra calificada respecto a los países desarrollados, pero si se le compara con otro conjunto de naciones, como China, India y Pakistán (y todos los que están en el mismo "cono de diversificación") probablemente tendría abundancia relativa de mano de obra calificada, con lo cual en realidad la apertura comercial habría beneficiado a este tipo de trabajadores. Otra explicación es que, contrario a lo que se esperaría siguiendo a la teoría convencional, México antes de la reforma comercial en realidad estaba protegiendo en mayor medida a las industrias más intensivas en mano de obra no calificada, de manera que la reducción de aranceles expuso a ese tipo de industria a la competencia internacional en mayor medida que a las industrias que utilizan mano de obra calificada. Por último, y en complemento de lo anterior, está el hecho de que junto a la liberalización comercial se abrió el país a la inversión extranjera, aumentaron las importaciones de bienes de capital y se reforzó el fenómeno de maquiladoras y el outsourcing por parte de compañías multinacionales, todo lo cual abonó en favor de la producción de bienes con mayor intensidad relativa en el uso del capital y de la mano de obra calificada Así, por estas vías se pudo haber influido en el alza relativa del salario de los trabajadores más calificados, aumentando la desigualdad salarial. 
Para apreciar si dentro del mecanismo de transmisión actuó el cambio en la proporción de empleados por tipo de calificación, se utilizó como variable dependiente la razón de empleados calificados en relación con los no calificados (resu), no encontrándose el cambio esperado de dicha variable, ni influencia de las variables del primer grupo, ni las relacionadas con la apertura externa y los precios relativos.

\section{Conclusiones}

Para el caso de México, los efectos de la liberalización comercial sobre el mercado de trabajo sólo pueden entenderse en el contexto complejo en que se desarrollaron. Uno de los cambios importantes observados en el periodo de la apertura externa que va de 1984 a 2002 es la clara tendencia al aumento de la desigualdad salarial. El retorno a la educación y el premio salarial a las ocupaciones que requieren mayor calificación tendieron a crecer en el periodo analizado, aumentando con ello la dispersión salarial.

Compatible con la creciente actividad económica en el norte del país, se observó una tendencia a elevar las remuneraciones relativas en esta región, donde se ha concentrado una parte importante de la inversión extranjera directa y con mayor orientación a la exportación. Por sectores productivos también se destacó la tendencia al aumento de la dispersión salarial, en especial en aquéllos con las mismas características de estar enfocados al exterior y ser receptores de inversión extranjera.

Durante el periodo analizado de la liberalización comercial, en el país se presentaron cambios en los precios relativos, tal y como lo predice la teoría convencional. Sin embargo, el patrón de protección comercial antes de la liberalización muestra más protegidas a las industrias con menor intensidad de capital, contrario a lo que predice la teoría. Así, la liberalización comercial tendió a modificar los precios relativos, haciendo bajar los más altos y subir los más bajos. El primer caso correspondió a industrias con menor intensidad del capital y el segundo a aquéllas con mayor relación capital-trabajo.

Se puede concluir que en el caso mexicano la liberalización comercial no implicó una recomposición drástica en el patrón de especialización productiva, en el sentido pronosticado por la teoría convencional. Esta situación puede estar asociada a que el país tiene ventajas comparativas en bienes intensivos en capital y en mano de obra calificada en relación con el conjunto de países en desarrollo; a la presencia importante de la inversión extranjera, dada la complementariedad capital-trabajo calificado, y al fenómeno de las maquiladoras. En consecuencia, la liberalización, si bien afectó claramente la dispersión salarial, su efecto sobre el empleo relativo esperado no es 
tan evidente, debido al cambio en los precios relativos que hizo reducir la renta de los sectores inicialmente más protegidos y de menor intensidad de capital. Por supuesto, es probable que además de la apertura externa otros factores hubiesen influido también sobre la desigualdad salarial, tales como la contención salarial, el nivel de sobrevaluación de la moneda mexicana y los cambios en el mercado de trabajo, que se observaron en buena parte del periodo analizado.

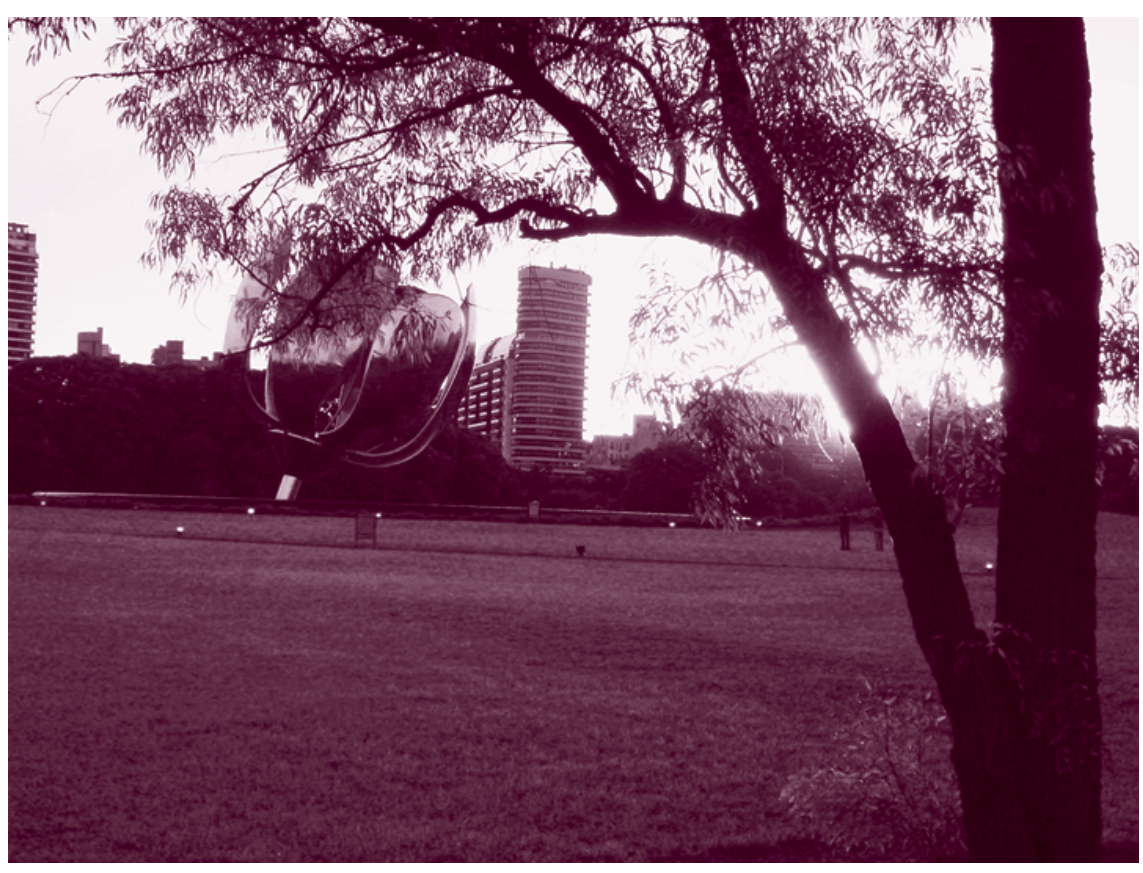

La flor II. Buenos Aires, verano 2005-2006. PAI 


\section{Bibliografía}

Attanasio, Orazio, Pinelopi Goldberg y Nina Pavchick, "Trade reforms wage inequality in Colombia", Journal of Development Economics, vol. 74, 2003, pp.1-32.

Berman, Eli, John Bound y Stephen Machin, "Implication of skill-biased technological change: international evidence", Working Paper Series, núm. 6166, Cambridge, NBER, 1997.

Burgos, Benjamín, "Efectos de la apertura externa sobre el mercado laboral de la región noroeste de México", tesis de doctorado en Ciencias Económicas, Tijuana, UABC, 2005.

Cragg, Michael y Mario Epelbaum, "Why is wage dispersion growing in Mexico? Is it the incidence of reforms on the growing demand for skills?", Journal of Development Economics, núm. 59, vol. 51/1, 1996.

Davis, Donald, "Trade liberalization and income distribution", Working Paper Series, núm. 5963, Cambridge, NBER, 1996.

Feliciano, Zadia, "Workers and trade liberalization: The impact of trade reforms in Mexico on wages and employment", Industrial and Labor Relations Reviews, núm. 55, 2001.

Fedderke, Johannes, Shin Yongcheal y P. Vaze, "Trade and labor usage: an examination of Stolper-Samuelson theorem for the south african manufacturing industry", Edinburgh School of Economics, Discussion Paper, Edinburgh, 1999.

Feestra, Robert y Gordon Hanson, "Foreign direct investment and relative wages: evidence from Mexico's maquiladoras", Working Paper Series, núm. 5122, Cambridge, NBER ,1995.

Galiani, Sebastian y Pablo Sanguinetti, "The impact of trade liberalization on wage inequality”, Working Papers Series, núm. 5424, Cambridge, NBER, 2003.

Green, Francis, Andy Dickerson y Jorge Saba, A picture of wage inequality and the allocation of labor through a period of trade liberalization: the case of Brazil, Canterbury, Departments of Economics, University of Kent, 2000.

Grilinches Z., "Capital-skill complementary", Review of Economics and Statistics, núm. 51/4, pp.485-488, 1969.

Hanson, Gordon, "What has happened to wages in Mexico since NAFTA? Implications for hemispheric free trade", Working Paper Series, núm. 9563, Cambridge, NBER, 2003.

Hanson, Gordon y Ann Harrison, "Trade liberalization and wage inequality in Mexico", Industrial \& Labor Relations Review, núm. 52-2, Nueva York, 1999.

Leamer, Edward, "In search of Stolper-Samuelson linkages between international trade and lower wages", Working Paper Series, núm. 5427, Cambridge, NBER, 1996.

López-Acevedo, Gladys, "Wages and productivity in mexican manufacturing", World Bank Policy Research Working Paper, núm. 2964, World Bank, 2003.

Mishra P., y Kumar U., "Trade liberalization and wage inequality: evidence from India", IMF Working Paper WP/05/20, 2005.

Pacheco-López, Penélope, The impact of trade liberalization on exports, imports, the balance of payments and growth: The case of Mexico, Canterbury, Department of Economics, University of Kent, 2004.

y Anthony Thirlwall, Trade liberalization in México: rhetoric and reality, Canterbury, Department of Economics, University of Kent, 2002.

Revenga, Ana, "Employment and wage effects of trade liberalization. The case of Mexican manufacturing", World Bank Policy Research Working Paper, núm. 1524, World Bank, 1995.

Robbins, D., "Worsening relative wage dispersion in Chile during trade liberalization, and its causes: is supply all fault", Harvard Development Discussion Papers, núm. 484, 1994.

Saba, Jorge, Andy Dickerson y Francis Green, "Trade liberalization and wages in developing countries", The Economic Journal, núm. 114, pp. F73-F96, Blackwell Publishing Oxford, 2004.

Trade liberalization and labor market in developing countries: theory and evidence, Canterbury, Department of Economics, University of Kent, 2001.

Stolper, Wolfang y Paul Samuelson, "Protection and real wages", Review of Economics Studies, núm. 9/1, pp.58-73, 1941.

Wood, A., North-South trade, employment and inequality. Changing fortunes in skill-driven word, Oxford Clarendon Press, 1994. 